\title{
La dialéctica andalucismo/socialismo en el contexto del sistema de partidos de la Transición. La emergencia nacionalista y la asunción estratégica de sus aportaciones por el PSOE (1977-1982)
}

The dialectics of Andalusism/Socialism in the context of the Spanish 'Transition' period party system. The emergence of nationalism and the Spanish Socialist Party ("PSOE") strategic appropriation of its contributions (1977-1982)

\author{
Manuel Ruiz Romero \\ Universidad de Sevilla \\ mruizromero@ono.com \\ ORCID: 0000-0001-5706-7802
}

Recibido: 25-1-2018

Aceptado: 16-7-2018

Cómo citar este artículo / Citation: RUIZ ROMERO, Manuel (2018). La dialéctica andalucismo/socialismo en el contexto del sistema de partidos de la Transición. La emergencia nacionalista y la asunción estratégica de sus aportaciones por el PSOE (1977-1982). Pasado y Memoria. Revista de Historia Contemporánea, 17, pp. 81-102. https://doi.org/10.14198/PASADO2018.17.03

\section{Resumen}

El presente trabajo analiza la puja político-electoral en el campo ideológico del socialismo democrático, entre un emergente y novedoso andalucismo político (Partido Socialista de Andalucía, PSA) y un histórico Partido Socialista Obrero Español (PSOE). Esta confrontación, nacida en los últimos años del franquismo en el ámbito de la oposición a la dictadura de Franco, es examinada en el contexto de la restauración democrática y en el marco de la conquista del autogobierno andaluz por la vía constitucional del máximo nivel competencial (artículo 151).

Palabras clave: Transición. Socialismo. Nacionalismo. Autonomía. Nacionalismo andaluz. Andalucismo. Partidos políticos. 


\begin{abstract}
This work analyses the political-electoral bid, within the ideological field of democratic socialism, between an emerging, novel Andalusian political party (Socialist Party of Andalusia, PSA) and a historical Spanish Socialist Workers' Party (PSOE). This confrontation, born in the last years of Francoism among Franco's dictatorship dissidents, is examined in the context of the democratic restoration within the framework of the conquest of Andalusian self-government via the Constitution highest level of competency (article 151).
\end{abstract}

Keywords: Transition Period. Socialism. Nationalism. Autonomy. Andalusian nationalism. Political parties.

\title{
1. Introducción
}

Andalucía no es una tradicional una comunidad destacada por sus reivindicaciones identitarias. En su seno ha venido propiciando un debate, conocido historiográficamente como Andalucismo Histórico que, desde la reflexión teórica que encarna la Constitución de Antequera de 1883 hasta el intento autonomista abortado por el 18 de julio con al asesinato de Blas Infante, transita desde limitados círculos políticos e intelectuales regeneracionistas hasta ser asumidas por las instituciones republicanas. Por ello, sorprende la rapidez con la que la ciudadanía andaluza cambia de opinión ante la necesidad de un autogobierno regional como reivindicación que adquiere más tarde un carácter cuasi nacionalista. ¿Simple mimetismo con otros territorios?, ¿Agravio comparativo?, ¿Sólo por el subdesarrollo secular que padece? De ser así de simple, entonces, ¿ipor qué no irrumpe con esa intensidad en otros territorios con igual o más necesidad de desarrollo?

A nuestro entender, existen varios factores que explican la emergencia de una conciencia regional antes, incluso, de la llegada de la Transición. Características que se complementan sin orden de relevancia alguno y cuyo resultado es el rechazo a cualquier situación de inferioridad o discriminación en los albores del reformismo. Del aludido subdesarrollo económico como elemento común a las ocho provincias, referimos muy especialmente los indicadores de emigración que el desarrollismo provoca y, con ello, la toma de conciencia de unas clases medias y populares que perciben nuevas posibilidades de progreso a la muerte de Franco. Los déficits estadísticos en los principales indicadores de calidad de vida de los diferentes territorios de Andalucía invitan a una respuesta colectiva y una estrategia común.

En el contexto de las singularidades apuntadas que identifican el discurrir de la transición en Andalucía, intentaremos en nuestro análisis adentrarnos en el origen, pormenores y desarrollo de la rivalidad política existente entre organizaciones (PSA y PSOE) que comparten un territorio un mismo espacio 
ideológico y electoral socialista. Si bien esa puja es legítima e intrínsecamente vital a todo contexto democrático representativo basado en la competencia entre una pluralidad de opciones, no es menos cierto que, para ambas siglas, el discurrir de esos años representa un devenir muy diferente y particular. Característico en Andalucía por único con respecto a otros territorios en el caso de la organización de Pablo Iglesias, e inédito por cuanto la irrupción sorpresiva del andalucismo político por vez primera en la historia llegando a tener Grupo Parlamentario propio en Cortes. Aún dentro de las aspiraciones de las fuerzas políticas por convertirse en hegemónicas, errores y aciertos de cada una de ellas se complementan con situaciones inéditas desde una perspectiva de Estado.

La copiosa bibliografía sobre todo del profesor Mateos (2017) y más recientemente las clarificadoras tesis de López (2011) y Rodriguez-Flores (2017), ponen de manifiesto en importantes síntesis de parciales trabajos anteriores, la adaptación de la histórica organización estatal socialista a un Estado descentralizado desde pretéritas posiciones centralistas, sin dejar atrás aspiraciones federales. En paralelo a estudios sobre su ideología y relato orgánico, el siglo XX ha sido todo un reto para el PSOE en cuanto al tránsito que las décadas representan y, con ellas su evolución por escenarios de muy distinto calado en referencia al debate territorial. Ahí están los estudios de los profesores Geniola (2017), Gillespie (1991), Mateos (1996), Méndez (2000), Molina (2015), Tezanos (2003), De la fuente (2017) y Santos (1996). Destacando para el ámbito andaluz los estudios de Caro $(2013 ; 2015)$ y Ruiz Romero (2003). De otra parte, la presencia del andalucismo político en el contexto de la transición ha sido del mismo modo objeto de importantes trabajos por parte de autores como Teba (1981), Rodriguez de la Borbolla (1986) y Ruiz Romero (2000b; 2002). No se queda atrás la singularidad del caso andaluz en el proceso de descentralización territorial abordado por los profesores Ruiz Robledo (1988; 2003), Ruiz Romero (2008), Hijano y Ruiz (2001); realidad jurídica que es completada por publicaciones y tesis sobre la aparición de las instituciones pre y autonómicas Ruiz Romero (2000a; 2005), Cruz (2017) y Santos (2002). Unos y otros han apuntado la fragilidad de las organizaciones políticas en Andalucía por el gran impacto que representa el devenir del proceso hacia la autonomía. Es el caso de García (2001), Ruiz Romero (2004b), Santos Silva (2014) y Soto (2015).

Dicho esto, nunca se ha explorado ni analizado el origen, fluir y resultantes de una rivalidad en no pocos casos aderezada de una cierta crudeza política y lejanía personal impropia del consenso imperante a lo largo del periodo constituyente. Nuestro objetivo con este trabajo es analizar en el arco temporal apun- 
tado, las claves que explican la rivalidad estratégica e ideológica entre PSA y PSOE a partir de uno de los legados más importante del antifranquismo: la reorganización territorial del Estado. Es sabido que la conjunción entre la izquierda parlamentaria y la presencia de nacionalismos subestatales no sólo representa un elemento central en dicha lucha sino que, en buena medida, incentiva la emergencia de regionalismos como el andaluz. Y es en el escenario constituyente donde la dirección socialista no sólo por estrategia sino por propia evolución, se adapta con más dificultad a una decidida dinámica descentralizadora en función de los avatares de la política en cada uno de los territorios. Con nuestro trabajo aportamos luz a los hechos, estrategias y discursos con la hipótesis de que para el PSOE, Andalucía fue una ámbito territorial que le aúpa por un lado al Gobierno y, por otro, deja libre de nuevas presencias nacionalistas el camino hacia una racionalización autonómica y la culminación del Estado de las Autonomías.

\section{El efímero despuntar de un sistema de partidos propios}

Ese regionalismo incipiente reclama igualdad de trato en un instante histórico muy determinado y, se perfila ideológica y electoralmente mediante nuevas formaciones de exclusiva obediencia andaluza que dibujan la posibilidad -frustrada más tarde como veremos- de un sistema de partidos diferencial. Aparecerán los siguientes grupos: Partido Social Liberal Andaluz (PSLA) de centro moderado que acaba integrado en UCD; Partido Socialista de Andalucía (PSA) un socialismo de izquierda que vive su edad de oro en los años del proceso estatutario, así como el Partido del Trabajo de Andalucía (PTA), vinculado a la izquierda marxista el cual, en parte, acaba tras la conquista autonómica integrado en el andalucismo político antes citado.

Lo cierto es que Andalucía, como sujeto político y unidad territorial, antes de estos factores apuntados no existe en atención de las arbitrarias divisiones a la que es sometida desde las administraciones del franquismo y, en base además, a la perversa asunción de su identidad prostituida por el régimen como referente del nacionalismo español ${ }^{1}$. No debe despreciarse tampoco la presencia de un regionalismo tecnocrático, el cual adquiere conciencia de la pobreza andaluza y de su potencial riqueza impulsando un Ente Regional Andaluz desde las Diputaciones franquistas entendido como una mancomunidad técnico administrativa ${ }^{2}$. Sectores intelectuales, universitarios y culturales pro-

${ }^{1}$ Sobre la comprensión de la historia y las identidades nacionales socializadas: Cfr. (Boyd, 2000; Archilés, 2015; Ortiz, 2009).

${ }^{2}$ Sobre ese incipiente regionalismo andaluz durante el tardofranquismo (García, 2001; Ruiz, 2000a; 2008; Saz; Archillés, 2011). 
mueven además en ámbitos artísticos, académicos o reivindicativos un mensaje de afirmación, disidencia y autoestima colectiva profusamente socializados desde numerosas iniciativas comunicativas que le prestan especial atención. Con ello, la aceptación de símbolos identitarios antes desconocidos (escudo, himno, bandera), la recuperación de hitos, personajes y doctrinas prohibidas como Blas Infante (1885-1936) y, por otro lado, una profunda reflexión política hacia las posibilidades de progreso y avance necesarias en una democracia. ${ }^{3}$

Todos estos elementos posibilitan un discurso diferencial rápidamente asumido y ansiosamente consumido, que se visualiza en las populosas e inesperadas manifestaciones del primer Día de Andalucía (4-XII-1977). Importantes, no tanto por su número (alrededor de dos millones de personas en el territorio andaluz dentro y fuera del Estado), como por lo que representa para unas élites políticas que sólo priorizan autogobierno político para territorios así reconocidos durante la II República. El asesinato, nunca aclarado, de Manuel José García Caparrós en Málaga durante una convocatoria festiva y familiar, alerta sobre la posibilidad del fácil recurso a la violencia en una sociedad depauperada donde despunta una ciudadanía con rasgos y discursos inéditos en su historia reciente. ${ }^{4}$

Consideramos que desde ese instante y durante lo que hemos denominado sexenio autonómico (1977-1982), una vez constituida la Asamblea de Parlamentarios Andaluces (octubre de 1977) hasta el primer parlamento autonómico, se inicia una competición política por liderar y capitalizar en las instituciones esa demanda reclamada en la calle. Hasta esos instantes, la rivalidad que como comentaremos, queda limitada a núcleos de oposición, adquiere ahora intensidad (potencial electoral) en el escenario del proceso al autogobierno. Se proyecta no sólo en las estrategias de cara a la conquista y gestión de la institución preautonómicas, sino en las maniobras políticas y discursivas internas de un mismo escenario socialista y, en este sentido, nos interesa retroceder en el tiempo para explicarnos mejor el momento, la dinámica y los hechos. ${ }^{5}$

El andalucismo político surge a principios de 1970 bajo la coartada legal de una sociedad anónima (CEPESA) al hilo de la presentación en 1966 de Alejandro Rojas Marcos al Ayuntamiento de Sevilla por el tercio familiar y su posterior condena a destierro de la ciudad al reclamar elecciones libres. Más adelante, su Manifiesto Fundacional, ya como Alianza Socialista de Andalucía

${ }^{3}$ Un completo repertorio sobre la misma en:<http://fundacionblasinfante.org/reposi torio-bibliografico-sobre-el-andalucismo-historico/>

${ }^{4}$ Como compilación documental sobre la cuestión: (Ruiz Robledo, 2003; Hijano; Ruiz, 2001).

${ }^{5}$ Una aproximación a la transición andaluza en (Santos, 2002; Cruz, 2017; Soto, 2015; Ruiz, 2005). 
(ASA), ve la luz en París (1973) en el seno de la revista Cuadernos de Ruedo Ibérico como iniciativa de oposición ${ }^{6}$. Su discurso aireaba la contradicción de una tierra rica que padece las consecuencias de un colonialismo interior que la condena a un subdesarrollo permanente inmerso en una identidad prostituida que la convierte en la "discoteca de Europa".

Es en octubre de 1975 cuando, marcando ya distancias con su texto inicial, vincularán la ruptura democrática con la recuperación de un autogobierno que la historia le niega el 18 de julio de 1936. Con el título Por un Poder Andaluz se proclama heredera de aquel movimiento emprendido en tiempos de la República, asume la doctrina de Blas Infante y proclama ser la única fuerza exclusivamente andaluza y socialista. En mayo de 1976, ASA hace pública su primera propuesta estatutaria sobre los acuerdos de la Asamblea Regional Andaluza de 1933 retomados por el Frente Popular en junio de 1936. Al paso de los años la ideología del andalucismo se definirá como nacionalista siendo legalizado como Partido Socialista de Andalucía (PSA). ${ }^{7}$

De otra parte, y compartiendo ese mismo espacio ideológico y electoral, el PSOE en 1974 y en su XIII Congreso en el exilio (Suresnes) en su Resolución sobre las Nacionalidades Ibéricas, aboga por primera y única vez en su historia a favor del derecho a la autodeterminación para las nacionalidades del Estado dentro de una Republica de clase. Reconociendo dicha posibilidad a "otras regiones diferenciales" sin llegar a concretar ${ }^{8}$. Es presumible que esa apuesta genérica y abierta, lo fuera ante un intento por superar desde la izquierda las posiciones más mesuradas que el Partido Comunista de España (PCE) había abrazado con anterioridad. En ellas, el principal partido de la oposición reconoce el derecho a decidir de las tres nacionalidades históricas que votaron sus estatutos y fueron reprimidas por el golpe militar, aunque, no es menos cierto que la vertebración territorial ocupaba un espacio marginal en las reivindicaciones de los sectores de oposición. ${ }^{9}$

Esta generalización y el radicalismo verbal son matizados en el XXVII Congreso del PSOE (diciembre de 1976), el primero celebrado en España tras la asonada, al respaldarse la existencia de "nacionalidades y regionalidades" dentro de un Estado federal, fórmula que poco a poco se aproxima al boceto cons-

${ }^{6}$ Cfr. (Alianza Socialista de Andalucía, 1973).

${ }^{7}$ Un análisis de su órgano portavoz (Ruiz, 2000b).

${ }^{8}$ Entiéndase su XXVI Congreso desde su nacimiento. Los estudios sobre esta organización son abundantes y copiosos. Citamos pues dos estudios ya clásicos: (Santos, 1996; Gillespie, 1991).

9 Así se hace constar en el informe del Secretario General Santiago Carillo al VIII Congreso celebrado en 1972 (Carrillo; Ibárruri, 1972: 44-46). 
titucional. Una organización tradicionalmente jacobina y que había cuestionado la aspiración autonómica de la Andalucía republicana por burguesa e innecesaria, se adaptará así paulatinamente a un periodo donde la descentralización del Estado es paralela al proceso democratizador ${ }^{10}$. Para ello, junto a su consolidación e implantación por la geografía andaluza, plantea una estrategia de unidad socialista con dos objetivos fundamentales: reforzar su organización y esclarecer el panorama electoral despejando el espacio ideológico del socialismo que debe ser identificado en exclusiva con sus siglas ${ }^{11}$. Sus notables resultados en las constituyentes de 1977 confirman esta necesidad táctica, toda vez que le confirman como primer partido de la oposición. El PSOE vive así su particular transición dentro de la transición, en la medida que entre 1968 y 1978 existen al menos cinco nuevos grupos dentro del mismo ámbito. Entre ellas, el PSOE-histórico, el Partido Socialista Popular (PSP) de Tierno Galván y la Federación de Partidos Socialistas -a la que se vincula el PSA- como referentes más significativos. ${ }^{12}$

\section{La oposición al franquismo como origen de una rivalidad política}

Si bien las constituyentes marcan en 1977 amplias distancias en la confianza que la ciudadanía otorga a cada formación, mayoritariamente en la escena andaluza a favor de posiciones de izquierda, es necesario indagar antes para localizar el origen de lo que es un constante y enconado enfrentamiento político en un territorio que resulta ser el único que conquista un autogobierno de primer orden a través del procedimiento "excepcional" de la Carta Magna. En este singular y complejo devenir pro estatutario es el escenario donde se desarrolla dicha cruzada estratégica e ideológica.

El reconocimiento de Alianza Socialista de Andalucía (ASA, embrión más tarde del PSA) entre la oposición antifranquista, se materializa con su presentación en París (marzo de 1974) junto a la Junta Democrática de España que lidera el PCE. Tiempo después, el PSOE crea la Plataforma de Convergencia Democrática con otros grupos a su izquierda. Fusionados ambos foros

${ }^{10}$ Así lo reclama una contundente editorial de El Socialista: "Los problemas artificiales. Un poco de formalidad señores" de 6 noviembre de 1932. El texto dirigido contra la iniciativa andaluza se burla de unas intenciones a las que califica de "desafortunadas", considerando que "carecen de sentido" los esfuerzos. Llama "mito" a la reivindicación en la medida que la define como "pasatiempos de señoritos", augurándole el mayor de los fracasos toda vez que sólo se siente la cuestión en Cataluña y País Vasco. Y sentencia: "Estatuto no".

${ }^{11}$ Cfr. (Mateos, 1996). Sobre la construcción y primeros años del socialismo andaluz (Rodríguez, 1986; Méndez, 2000; Caro, 2015; 2013; Ruiz Romero, 2003).

12 Sobre estas siglas, su nacimiento y el proceso de integración en el PSOE (Rubio, 1996; PSP, 1977; Bobillo, 1976). 
-Platajunta-ASA se autoexcluye de la Coordinadora Democrática de Andalucía al considerar que no se reconoce al territorio andaluz como sujeto político: ni con los mismos derechos, ni mismo trato igualitario con otros históricos. Esta reproducción "sucursalista" de la izquierda tradicional colisiona con la percepción de un partido andaluz que aspira a consolidarse en ese espacio electoral propio.

Las posiciones se enconan aún más cuando se crea la Federación de Partidos Socialistas (1976) formada por once grupos, entre ellos, el Partido Socialista de Andalucia una vez deja atrás las siglas ASA en su I Congreso $(1976)^{13}$. Es más, esta distancia programática y estratégica, se refuerza cuanto la citada Federación concurre en 1977 a las constituyentes coaligada con el PSP de Tierno Galván bajo las siglas Unidad Socialista (US). Los resultados despejan al escenario andaluz: el PSOE logra la mayoría: 27 diputados y 14 senadores, frente a un parlamentario por dicha coalición, el cual, finalmente, se integra en el PSOE por mor de la exitosa estrategia de este último en pro de la citada unidad socialista.

Tras las primeras elecciones, constituida la Asamblea de Parlamentarios de donde está ausente el andalucismo, la disputa es fundamentalmente entre UCD y PSOE alrededor de la creación del primer gobierno de un ente preautonómico con personalidad jurídica propia (Junta de Andalucía). Sin embargo, en 1979 la sorpresiva irrupción del PSA en la I Legislatura (cinco diputados cuando las encuestas apuntaban uno) formando grupo parlamentario propio y los significativos resultados obtenidos en el escenario local, parecen motivados por una estrategia discursiva basada en denunciar la discriminación que otorga a Andalucía el Título VIII de la Constitución y de la cual se culpa al bipartidismo centralista. Cuestión ésta que imprime singularidad y definición al voto de un andalucismo político que en su II Congreso se define ya como nacionalista (enero 1979). De otra parte, no hay que olvidar que, aplicando la ley de D'Hondt los cinco escaños alcanzados entonces por el andalucismo en dicha convocatoria representan la pérdida de 10 diputados para el PSOE andaluz. Importante contratiempo que no podía permitirse una fuerza política que aspira a convertirse en alternativa en la Moncloa. Es más, Euskadi y Andalucía, donde la irrupción de fuerzas nacionalistas es más contundentes, representan dos serios lastres dado que el PSOE lidera sus órganos preautonómicos y habían incidido más sobre cuestiones sociales y solidarias que territoriales. ${ }^{14}$

\footnotetext{
${ }^{13} \mathrm{Al}$ respecto: (Barón, 1976).

${ }^{14}$ Todo indica que aprobada la Constitución, el regionalismo latente de las preautonomías emerge electoralmente para traducirse en una singular irrupción de formaciones nacionalistas en Cortes. A los dos territorios apuntados puede sumarse también Canarias.
} 
Los intentos ampliamente testimoniados por integrar las siglas andalucistas en el PSOE fracasan finalmente. Así, en tanto ambas formaciones comparten el mismo espacio electoral; ante la irrupción del andalucismo limitando la presencia del PSOE en Cortes; siendo el PSA el único grupo de la Federación de Partidos Socialistas que no se integra; fracasados los intentos del PSOE por liderar el proceso al autogobierno, ¿qué restaba por hacer? ${ }^{15}$. Radicalizar su discurso autonomista. Asumir tácticamente y de forma improvisada un andalucismo social a través de un dirigente que cree en él, Rafael Escuredo, situándole al frente del segundo gobierno preautonómico (y primero autonómico). Ante la ascendencia andaluza de los líderes del PSOE, se acentúa así desde su federación más importante un andalucismo táctico que arrebata banderas discursivas al PSA mostrando su nítida voluntad de autogobierno ante las dificultades (o torpezas del ejecutivo centrista). El caso andaluz sirve de ejemplo para que la organización muestre su amplia voluntad descentralizadora, convirtiéndolo en una experiencia única en el Estado. Su éxito le impulsa a la conquista del Gobierno Central visualizando las contradicciones del gobierno Suárez en dicho sentido, las debilidades estratégicas del nacionalismo andaluz e, interceptando el crecimiento a este último como nos disponemos a comentar. ${ }^{16}$

\section{El singular nacionalismo del Presidente Rafael Escuredo}

La estrategia es denominada en términos periodísticos como Escuredismo desde sus primeros momentos por causa de su protagonista. Definámosla como una respuesta puntual, calculada, limitada en el tiempo y exclusiva para Andalucía. Destinada por un lado a vencer a UCD, por otro, superar al PSA liderando el proceso autonómico en el seno de una política de acoso permanente del PSOE hacia ambas formaciones empleando un discurso más radical ${ }^{17}$. Al aparato central del PSOE le interesaba simular un mayor autonomismo andaluz tanto, como desactivarlo cuando gobernase Felipe González. Es más, Andalucía repre-

Cfr. una visión de conjunto sobre esta irrupción de formaciones regionalistas/nacionalistas: (Sánchez, 1979).

${ }^{15}$ Intentos comentado y documentado en: (Teba, 1981: 306 a 310).

${ }^{16}$ No en vano en su Memoria de Gestión a su II Congreso, el PSOE andaluz muestra su preocupación por el inesperado respaldo de los andaluces al PSA. Fuerza a la que consideraban interclasista, pequeño burguesa, sectaria y desideologizada, acusándola de ser un factor de inestabilidad en la aplicación de la Carta Magna. Se interpreta el respaldo a estas siglas a causa de una mera conciencia de pueblo oprimido sobre la marginación que sufren los andaluces, incentivada por simple agravio con otros territorios. En ningún caso, como una toma de conciencia nacional. Cfr. (Federación Socialista de Andalucía, 1979: 8).

${ }^{17}$ En: (Santos, 2002: 281). 
senta un singular relato convertido en problema de Estado en cuanto a las dificultades de sus avances descentralizadores y, como señalan algunos de sus biógrafos, es aquí donde comienza del declive de Adolfo Suárez una vez tiene lugar un sustancial empuje que acerca el socialismo al Ejecutivo central.

La estrategia se desarrolla siendo conscientes de las diferentes tácticas seguidas desde la dirección del PSOE respecto a Andalucía y en cuanto la descentralización de los territorios. Dentro de los que consideramos una profunda convicción personal hacia un autogobierno pleno, todo gira alrededor de Rafael Escuredo. Su singular posición la había dado a conocer ante el órgano portavoz de su partido: El Socialista. Mediante entrevista, el primer titular de la Junta de Andalucía deja clara su percepción, consecuente a su entender, con un pensamiento de clase y marxista del socialismo. Según él, existen "dos clases de Andalucía (...) una conservadora con concepción clasista, y otra progresista que se debe definir sólo y exclusivamente como andalucismo de clase. En este sentido, un proyecto político para Andalucía pasa por la autonomía. Según él, aquí hemos pasado de un regionalismo andaluz a un inicial nacionalismo que unos traducen en votos y otros en vivas a Andalucía en Madrid"18.

Su liderazgo personal en Andalucía explica que durante el II Congreso del PSOE-A (diciembre 1979) Escuredo intente imitar al PSC catalán o al PSE vasco, y apueste por impulsar una organización andaluza federada al PSOE bajo parámetros de una "nacionalismo de clase". De hecho, así llega a definirse: "Soy un nacionalista andaluz" dirá ante los medios ${ }^{19}$. Derrotadas sus posiciones dejará de representar los intereses de su partido para convertirse en un

${ }^{18}$ Entrevista a Escuredo por Francisco Vélez, El Socialista, 112, 10-VI-1979, p. 6. Las referencias a los vivas a Andalucía en Madrid, aluden al final de las intervenciones del Grupo Parlamentario Andalucista en Cortes, las cuales finalizan con un "Viva Andalucía Libre", recordando las últimas palabras de Blas Infante al caer fusilado por el golpismo días después del 18 de julio.

${ }^{19}$ Se muestra consciente de la polémica que puede motivar cuando señala: "fuera de mi partido pueden crear cierta preocupación, en la medida que algunos crean que el nacionalismo es un patrimonio exclusivo inscrito a su nombre en el registro de la propiedad". El Correo de Andalucía, 9-VI-1979, pp. 1 y 17. En este mismo medio y día, el mismo comentarista José Álvarez apunta algunas pautas de cómo puede entenderse las declaraciones de Escuredo en el PSOE: "está claro que Escuredo ha pensado que si Txiki Benegas o Raventós pueden ser nacionalistas en el País Vasco y en Cataluña por qué no lo iba a ser él en Andalucía”. Incluso, se pronuncia sobre la autenticidad de estas declaraciones: "pesamos que Escuredo se cree lo que dice. Alguna que otra vez hemos afirmado que en Andalucía hay dos personas que creen de verdad, con distancia sobre los demás, en la Junta de Andalucía y en el autogobierno andaluz. Manuel Clavero Arévalo, que es un poco el inventor de la criatura es uno de ellos. El otro Escuredo". Igualmente, anima al Presidente a recoger "las aspiraciones nacionalistas", lo cual pasa "por un partido menos dependiente de Madrid". No en vano apunta, "la línea 'escuredista" (...) se ha rebelado contra el aparato". 
Presidente de la Junta de Andalucía con un liderazgo reconocido popularmente y sobre el que se sustenta la astuta táctica. Significa por tanto, una anómala estética nacionalista dentro del socialismo en general y, particularmente en el andaluz, así como una completa reconsideración de la actitud estatal del PSOE a la hora de su política autonómica. No en vano la Resolución sobre las Autonomías confeccionada a raíz del XXVIII Congreso (mayo 1979) cita expresamente a Andalucía junto a Canarias, como territorios que aspiran a igualarse a otros históricos con una dimensión más nacionalista. La solución a los desequilibrios entre futuras comunidades Autónomas la representa una autonomía más social y solidaria y no tanto identitaria. A nadie escapa, por otra parte, que el debate encarna un importante reto interno y orgánico para una organización de ámbito estatal.

El Escuredismo asume así y lidera los tradicionales argumentos y estéticas del andalucismo ${ }^{20}$. Convive junto a un discurso de Estado y otro ajustado a las singularidades de cada territorio, mientras que la ortodoxia de su partido la representan otras voces y/o barones en sentido diferente. Cautos en no poco casos con el proceso de descentralización, además de propensos a pactos con el centrismo como preámbulo de lo que sería el primer gran pacto de Estado en la política territorial ${ }^{21}$. A diferencia del caso gallego, con la compleja integración del Partido Socialista de Galicia en el PSOE, se obtiene una alta rentabilidad electoral a partir de un posible conflicto interno, ofreciéndose además una contundente imagen de autoridad por parte del aparato de dicha organización. Todo indica también que, en otros territorios como el llamado País Valenciano o Canarias tan legitimados para desarrollar el procedimiento al autogobierno como en el caso andaluz por el artículo 151 de la Constitución, el PSOE no lo tiene tan claro en la medida que sus opciones no son tan decidida-

${ }^{20}$ Precisamente, hasta estos momentos, la figura de Blas Infante, nominada más tarde por el Estatuto de Autonomía en 1981 como Padre de la Patria Andaluza venía siendo reivindicar casi en exclusiva por las posiciones nacionalistas. Con Escuredo, forma una parte sustancial de su discurso reivindicativo y, junto a la figura del notario asesinado en 1936, otros tantos hitos, símbolos, personajes y discursos vinculados al Andalucismo Histórico. La bibliografía sobre estos antecedentes en: <http://fundacionblasinfante.org/repositoriobibliografico-sobre-el-andalucismo-historico/>

${ }^{21}$ No es casual que Alfonso Guerra, con una sensible dosis de ambigüedad, se pronunciase a favor de encaminar gran parte de las autonomías por el artículo 143 sin precisar cuáles no seguirán dicho procedimiento (El País, 6-XI-1979). De igual forma, son reiteradas las declaraciones de la Secretaria de Política Autonómica del PSOE, la diputada granadina María Izquierdo, insistiendo que con el 143 podrían alcanzarse los mismos contenidos que en el 151, si en un futuro así se decidiese. Tesis ambas cercanas a las que UCD defiende para el caso andaluz: único territorio donde el PSOE apuesta por el procedimiento del 151 para su autogobierno. 
mente identitarias. En ese escenario donde Andalucía se convierte en todo un símbolo y una apuesta estratégica para la partida de Estado de dicha organización. Vencer en las elecciones autonómicas de Andalucía representa para el PSOE estar más cerca de la Moncloa y, en esa lógica, tener un Presidente en dicha Comunidad nacionalista o pseudo andalucista, significaba un lastre demasiado peligroso por las contradicciones que representaba. ${ }^{22}$

Con la negativa por parte de UCD a un autogobierno vía 151 equiparable a otras nacionalidades, Escuredo abandera un radical autonomismo inédito en sus filas fuera de territorios históricos, de manera que alrededor de su persona y figura institucional gira la batalla del referéndum del 28F. Andalucía comienza durante el verano de 1979, el procedimiento para su acceso al autogobierno de máximo competencial por el artículo 151 de la Constitución con un abrumador respaldo por parte de las Corporaciones Locales. Fijada la fecha para el respaldo ciudadano a dicha vía, es justo en el interin donde la Ley de Referéndums está a punto de aparecer en el BOE cuando UCD cambia su posición y abraza la reconversión del proceso al autogobierno por el artículo 143 de la Carta Magna.

Llegados a este punto, es justo señalar además la presencia de errores tácticos, profusamente aireados de manera oportuna desde el PSOE hacia un PSA que no comunica bien a su electorado sus políticas. En primer lugar, el cambio de alcaldías durante el pacto de izquierdas tras las primeras municipales; en segundo lugar, por su acuerdo con UCD para desbloquear el autogobierno mediante la fórmula del "interés nacional" (art. 144), posibilitando así la continuidad por el artículo 151 una vez bloqueado tras el fracaso del 28 de febrero de 1980. Entre otras cuestiones, por las rígidas condiciones de Ley de Referéndums pactada entre UCD y PSOE pese a las advertencias de los Grupos Parlamentarios Comunista y Andalucista los cuales, no respaldarán su aprobación en Cortes. ${ }^{23}$

${ }^{22}$ Compartimos el parecer del profesor Ruiz Robledo (1988:39) cuando afirma que de un primer enfrentamiento en la preautonomía entre UCD y PSOE, se pasa a una segunda etapa donde con Escuredo al frente del segundo gobierno preautonómico la rivalidad es entre PSA y el resto de partidos con representación parlamentaria.

${ }^{23}$ Algunos autores insisten en el hecho de que en vez de acudir al Decreto-Ley para resolver el desfase legal ordinario, tal y como se aplica en los casos de los refrendos de los articulados vasco y catalán, se recurre a la vía del desarrollo constitucional por el procedimiento de urgencia. Hecho que interpretan como "un instrumento de adaptación a los esquemas gubernamentales del futuro desarrollo autonómico, y no en el armazón jurídico integrador que en la Constitución se preveía". Dicho así, nuestra hipótesis es que este cambio de actitud representa el primer acercamiento político entre los dos partidos mayoritarios a la hora de un acuerdo de Estado que se concretará de cara a racionalizar las autonomías. Cfr. Así lo defienden (Linde; Herrero, 1980: 85-86). 
Tal y como se ha señalado, la consulta del 28 de febrero de 1980 fue convocada por el Gobierno para perderla. Quien no votara, en realidad, restaba porcentaje para solventar el referéndum. Cualquier error, abstención o dato no actualizado del censo, pondría más cuesta arriba superarlo. Pese a las características geopolíticas de Andalucía, el respaldo gubernamental a la consulta estuvo más cerca del boicot: los medios de comunicación del Estado fueron controlados por el centrismo y utilizados de forma limitada por la Junta, los censos municipales no estaban actualizados, aumentaron las dificultades del voto por correo, se recortaron tanto los días de campaña, como la publicidad institucional y los recursos económicos. La rabia se incrementa más aún al conocerse el enrevesado texto de una pregunta donde estaban ausentes las palabras autonomía y Andalucía. ${ }^{24}$

Sólo una gran movilización popular comparable a la del 4 diciembre de 1977 será capaz de hacer frente a unos condicionantes que fomentaban el desinterés, el desconcierto y la abstención. En protesta por esta actitud obstruccionista, el segundo Presidente de la preautonómica Junta, Escuredo, realiza una huelga de hambre por 72 horas (3-5 febrero de 1980). Durante la campaña se consuma la ruptura de la UCD en Andalucía donde una parte asume el 151 y respalda la dimisión de del Ministro Clavero Arévalo. Meses antes nadie podía adivinar esta polarización de posturas y la crispación alcanzada. ${ }^{25}$

Los medios gubernamentales anunciaban la noche electoral el fracaso de la consulta en Huelva, Córdoba, Málaga, Jaén y Almería; no obstante, tras el recuento y algunos recursos, en esta última provincia y por 22.000 votos, se produce la derrota jurídica, que no política, del 28 de febrero ${ }^{26}$. No se supera la consulta con la Ley de Referéndum y el procedimiento por el artículo 151 queda bloqueando. La confusión aún hoy existente es que aquella cita se salda con un resultado positivo, con lo que se elimina la batalla parlamentaria que vendrá,

${ }^{24}$ En concreto: “ ¿Da usted su acuerdo a la ratificación de la iniciativa prevista en el artículo ciento cincuenta y uno de la Constitución a efectos de la tramitación por el procedimiento establecido en dicho artículo?".

${ }^{25}$ Con seguridad, el ejemplo más expresivo estuvo en la familia del propio Presidente Escuredo, de forma que mientras él y su esposa figuraban como dados de baja en el censo, su hija de seis años aparecía de alta con 36 años. Sus declaraciones tras serle negado su derecho a voto pasaron a ser resumen de lo sucedido: "Andalucía es un país donde los muertos votan y los vivos tenemos problemas para hacerlo". El incidente, es ampliamente destacado en la prensa del día siguiente de la consulta, de forma que, tras serle emitido un documento por la Delegación de Estadística del Ayuntamiento, emite finalmente su voto.

26 Se emiten emite tres sentencias (80/1980, 81/1980 y 82/1980), referidas, respectivamente, a los recursos interpuestos para Almería, Granada y Jaén. Sin embargo, no acepta la repetición de la consulta en la primera de estas provincias. ABC, 13-IV-1980, p. 8. 
y se magnifica el significado de aquella fecha. Sólo conociendo esta limitación legal y la intensa movilización popular tras las urnas, puede reconocerse la diligente labor en Cortes de ingeniería parlamentaria en favor de un desbloqueo político. ${ }^{27}$

\section{El hito del referéndum del 28 de febrero}

El significado de la consulta del 28 de febrero de 1980 debe entenderse tanto como una derrota en términos jurídicos, como una importante victoria política de una Andalucía que en un amplio porcentaje expresa sus aspiraciones en favor de un autogobierno pleno. Sin embargo, lo sucedido significa un inusitado impulso político y movilizador ${ }^{28}$. Acto seguido, los andaluces de la mano de sus representantes democráticos en Cortes, se disponen a seguir dando para contar. El 28F no había concluido y aún con diferente intensidad en el seno de los partidos políticos, la batalla por el autogobierno dará mucho para contar. Bloqueada la vía "rápida", la respuesta ciudadana a las condiciones en las que se desarrolla la consulta, logra que la proyección política del hito adquiera una dimensión estatal en la medida que cuestiona y paraliza todo la descentralización territorial. No en vano, crisis económica y Estado autonómico son argumentos que, junto con la actitud del gobierno hacia el referéndum, son los ejes de la Moción de Censura contra el Presidente Suárez, la cual no prospera pese a estar apoyada por todos los parlamentarios andaluces de izquierdas (mayo de 1980).

En un principio, la situación provoca actitudes muy dispares. Mientras que el PSA reclama la retirada de las Cortes de los parlamentarios andaluces o la reforma del texto constitucional, el PCE muestra su desconcierto a la espera de soluciones políticas. A su vez, el PSOE se inclina ahora, algo probado mediante un cartel editado al efecto que nunca llega a circular, por el 143 que tanto criticó a UCD y respaldando ahora su perspectiva en los dos millones de votos afirmativos a la autonomía "más votada de España".

Con la Ley en la mano, Andalucía tiene cerrado el camino hacia una autonomía que la equipare con otras nacionalidades históricas. Pese a ello, la movilización popular y las sucesivas maniobras obstaculizadoras del Gobierno centrista ponen de manifiesto la victoria moral y política, de manera que, después de la fecha del plebiscito los partidos buscan respuestas parlamentarias para superar la situación. Existían tres caminos legales para desbloquear la

${ }^{27}$ Sobre la negativa de UCD. Cfr. El País, 5-III-1980. Esta misma cabecera en su editorial del día 7 de este mismo mes, bajo el título "Torpezas en Andalucía" analiza los resultados y subraya la respuesta inesperada de Andalucía a su referéndum "que no puede desdeñarse".

${ }^{28}$ Sobre los pormenores de la consulta (Ruiz, 2004a). 
situación: repetir la consulta en Almería atendiendo a los errores del censo; modificar los extremos de la Ley de Referéndums o reformar el artículo correspondiente de la Carta Magna y relajar -ahora sí- las condiciones del $151^{29}$. Esta última propuesta presentada simbólicamente por el Grupo Parlamentario Andalucista, queda sin efecto una vez el amplio concenso final que citaremos. La respuesta a esta parálisis inicial es el registro en el Congreso por parte del PSA de una iniciativa para repetir la votación en Almería. Al registro de la propuesta (12 de marzo 1980) le siguen días después el resto de formaciones en términos muy similares. Sustanciadas todas de forma conjunta son derrotadas en el Congreso por sólo un voto de diferencia (12 de junio 1980). La opción de repetir la consulta es abortada y, con ello, será plena la responsabilidad futura al respecto de las dos formaciones que analizamos.

Como respuesta a la Moción de Censura contra Suárez apoyada por todos los partidos andaluces, el partido que lo sustenta plantea un Debate de Confianza sobre su liderazgo (16-18 septiembre 1980). Es en este escenario donde conoce desde la misma tribuna del Congreso, un acuerdo político entre UCD y PSA para desbloquear la autonomía utilizando el "interés nacional" recogido en el artículo 144. Precepto éste que, ni representa un competencial político de autonomía, ni una devaluación de la misma como se hace valer desde las posiciones del PSOE. Se trata de una posibilidad política para solucionar la parálisis a través de un camino que evite, tanto la reforma de la Constitución como la repetición en Almería. En cualquier caso, reconociendo siempre la victoria política del 28F y conquistando la autonomía plena del 151.

Una vez se hace público el anuncio, todos los medios de comunicación reconocen la oportunidad de la propuesta, pero las fuerzas de oposición en la Junta donde PSOE y PCA tienen mayoría, mantienen su negativa a un acuerdo que les margina aunque aporte solución. Rechazo más por razones políticas que jurídicas, y para el que se emprende una intensa campaña mediática de desprestigio y confusión sobre el significado del acuerdo equiparando el artículo 144 con el nivel competencial del 143. El PSOE amenazará con recurrir al Tribunal Constitucional con lo que, según la normativa de dicha alta instancia judicial en aquel entonces vigente, la autonomía se habría paralizado a la espera de una sentencia que podría tardar años. De nuevo, el proceso

\footnotetext{
${ }^{29}$ En no poco instantes, la rivalidad política se convierte en ataques personales entre los líderes tanto de PSA como de PSOE. Baste como ejemplo el artículo que Peces-Barba publica en El País (13-VIII-1980) con el expresivo título de "Reflexiones sobre la conducta política de Rojas Marcos", y en cuyo contenido se apuntan algunas consideraciones biográficas y graves descalificaciones personales sobre el andalucista. Respondido a su vez en esta misma cabecera por José Aumente: “¿Qué pretende el PSOE?”, (21-VIII-1980, p. 8)
} 
corre el riego de seguir bloqueado. Pero algo se había movido en el escenario del 28F y de una manera arriesgada. El PSA había forzado una solución legal de la mano de una desacreditada UCD que, por deméritos propios, padece un amplio rechazo entre los andaluces. Un desenlace viable, que no supo, no pudo o no le dejaron explicar al andalucismo político. UCD en el escenario andaluz no puso ningún énfasis ni se movilizó más allá de lo justo para explicar la oportunidad, legalidad y viabilidad de un 144 que acaba por convertirse en impopular por desprestigiado, e identificado como el 143 entre el desconcierto, la desinformación y la manipulación interesada. Será el instante propicio utilizado desde el PSOE para descalificar al andalucismo acusándolo de traicionar Andalucía. Un punto de inflexión definitivo que resuelve la disputa entre las dos formaciones en favor de PSOE bajo la acusación de oscuros intereses hacia los nacionalistas andaluces.

Con el bloqueo prorrogado la posición de UCD se ve forzada al diálogo. A principios de octubre, tras meses de estéril enfrentamiento, Adolfo Suárez y Felipe González inician conversaciones para buscar un acuerdo. Se llega así a una solución, consensuada y firmada por todas las fuerzas políticas andaluzas con representación parlamentaria por la que un amplio consenso pone fin al conflicto político. Los Diputados y Senadores de Almería suscriben conjuntamente un escrito reclamando a las Cortes que: "por motivos de interés nacional,... se sustituya la iniciativa autonómica en la provincia de Almería", "al objeto de su incorporación al proceso autonómico de las otras provincias andaluzas" por el artículo 151. Los representantes de PCE, PSA, PSOE y UCD, en la misma fecha (23 de octubre de 1980), "declaran sustituida en esta provincia la iniciativa autonómica al objeto de que se incorpore al proceso autonómico". ${ }^{30}$

Ambos documentos, con una unanimidad que evita el recurso a la vía judicial, se basan en el "interés nacional" (aspecto éste -insistimos- sólo citado en el artículo 144 de la Constitución) y en el amplio consenso que suscitan, de manera que dan paso a una propuesta conjunta para modificar la Ley de

${ }^{30}$ BOCG, Congreso de los Diputados, 24-X-1980, núm.108-I, serie b, pp. 413-414. Registrada con número 5878 el 23 de octubre de 1980 y suscrita por los cuatro portavoces de los Grupos que la avalan según este Boletín. El original consultado en el Archivo del Congreso de los Diputados en la carpeta de la tramitación de esta Ley (12/80) constan ocho firmas que corresponden a: Félix Manuel Pérez Miyares, Miguel Herrero de Miñón, Santiago Carrillo, Fernando Soto, Alfonso Guerra, Luis Yánez, Miguel Angel Arredonda, y Alejandro Rojas Marcos.

La cuestión, como ha señalado la profesora Ripollés (1980: 313), no hace más que poner de manifiesto la premura con la que la LORDMR se elabora. No obstante, sostenemos nuestra hipótesis antes apuntada sobre un acuerdo político precedente de otros autonómicos que vendrán. 
Referéndum. En esta nueva versión de la norma posibilita la flexibilidad no admitida en su redacción por UCD y PSOE. De esta forma, las dos Leyes Orgánicas 12/80 y 13/80, ambas de 16 de diciembre representan, en el primero de los casos, la reforma de la Ley de Referéndums; en el segundo, la sustitución de los resultados del 28F en Almería por la demanda de sus parlamentarios.

Las primeras elecciones andaluzas actúan como primarias de Estado y su amplia mayoría socialista inicia un gobierno cuasi monocolor hasta el presente. La rivalidad mantenida se decanta definitivamente del lado de la organización histórica de Pablo Iglesias. Es más, a diferencia del ejemplo valenciano donde el procedimiento autonomista se reconvierte por el artículo 143, el socialismo justificaría la armonización del proceso autonómico tras el ejemplo andaluz por mor de la intentona golpista del 23F dejando patente una voluntad racionalizadora y solidaria entre autonomías. Apostando ahora más por una política de igualdad competencial entre territorios y procedimientos de accesos una vez la citada Ley de Referéndums, limita la continuidad del País Valenciano y Canarias por el mismo artículo 151, aunque garantizaba la existencia de normas específicas que suponen el mismo trato en cuanto a transferencias (LOTRAVA y LOTRACA). La significación electoral del PSOE en cada uno de estos territorios representaba un factor que posibilitaba este novedoso encauzamiento racionalizador ${ }^{31}$. Pese a las posiciones más moderadas que el PSOE sigue ante la Carta Magna, el caso andaluz es único en una perspectiva de Estado por cuanto la actitud de radicalización momentánea e intencionada que mantiene. Estrategia con la que se fuerza y culminan también acuerdos para la descentralización territorial entre UCD y PSOE ante el temor de que la implantación del Estado de las Autonomías desborde los límites constitucionales.

\section{Conclusiones}

En ningún caso, los defectos constitucionales que pueden alegarse para la fórmula empleada en el desbloqueo desmerecen la victoria política de los andaluces, el 28F y su empuje durante todo el proceso de la conquista del autogobierno. Más bien, como se ha dicho, estamos ante un ejemplo de cómo las leyes están sujetas a intereses políticos.

A nuestro juicio, si bien es excesivo atribuir toda la intensa movilización proautonomista de esos años a la existencia de un andalucismo político; no cabe duda que esta formación representa un efecto catalizador en su esfera política. De no haber existido esas siglas, esa inesperada expresión popular desde

${ }^{31}$ Un análisis crítico de todo el desarrollo del Título VIII de la Carta Magna y su posible papel en el debate territorial presente (Pérez, 2017). 
la sociedad civil hubiese tenido lugar igualmente como nutriente vital donde se sustenta toda la batalla autonómica Dicho de otra forma, esa inquietud electoral y de expectativas socio económicas, hubieron de encauzarse por la vía institucional y ello, junto a grandes dosis de perplejidad por su irrupción, convierten a ese liderazgo en un hecho trascendente para Andalucía y el Estado.

Verdaderamente, la intención PSOE no era tanto convertir a Andalucía una nacionalidad histórica (aunque en términos jurídico políticos lo acaba siendo); sino convertir el proceso andaluz en la punta de lanza para conquistar su hegemonía en el Estado. Las llamadas entre los dirigentes socialistas a la racionalización autonómica y su aplicación al iter andaluz, la armonización autonómica pactada con UCD, el que su autogobierno fuera el único y último del procedimiento del art. 151, la elevada conflictividad que alcanza su devenir en el Estado... son factores objetivos que, sumados al intento de golpe del 23F, justifica y demuestran el fin de una estrategia de recambio donde el Escuredismo garantiza el triunfo del PSOE en territorio andaluz y, con él, gran parte de su contundencia electoral en el Estado. Como bien se ha expresado: "El modelo que pactaron \{UCD y PSOE\} permitía la pluralidad pero diluyó cualquier aspiración nacionalista (...) se impusieron más cerrojos para salvaguardar la unidad de España"32.

En el análisis sincrónico de un partido de dimensión estatal como es el PSOE, encontramos en Andalucía un proceder inédito radicalizando su discurso regionalista hasta extremos de competir en estética y acción con las tesis nacionalistas. Institucionalizado el autogobierno y consolidada en el Parlamento autonómico una cómoda mayoría electoral, dicha estrategia se desactiva en beneficio de tesis más moderadas que priorizan la construcción del Estado de las Autonomías y el desarrollo de la Constitucional, que le empujan hacia la contundencia representativa que alcanza en las Cortes de 1982.

La doble derrota del PSA (primeras andaluzas y II Legislatura) supone la reducción del nacionalismo a un papel testimonial que le obliga a desprenderse de su dimensión socialista para convertirse finalmente, tras un tiempo de transición (PSA-PA), en Partido Andalucista (PA). Realidad que confirma la preponderancia de una organización como el PSOE y su victoria final tras años de enconada rivalidad mutua. Aun Escuredo respaldado con una amplia mayoría en la cámara andaluza, en base a sus posiciones andalucistas es obligado a dimitir por su propio partido de forma sorpresiva tiempo más tarde

32 Así lo entiende entre sus conclusiones (Rodríguez-Flores, 2017). En esta misma línea y en el análisis de dicha evolución posibilista interna: (De la Fuente, 2017; Tezanos, 2003; Molina, 2015; Geniola, 2017). 
$(1984)^{33}$. Desde entonces, la Junta de Andalucía y el autogobierno son concebidos bajo las percepciones de unas siglas que siguen gozando hoy de un amplio respaldo entre los andaluces. Es entonces cuando se procede por parte de un PSOE a la desactivación intencionada de esa conciencia popular movilizada proto nacionalista que se logra durante el sexenio comentado, y que cuenta con contundentes respaldos tanto en el Gobierno Central como en las primeras legislaturas autonómicas. Realidad que encarna el fracaso de un singular sistema de partidos en Andalucía y, en paralelo, la firme consolidación del PSOE-A al frente de su autogobierno junto a una paulatina languidez electoral y orgánica del andalucismo político hasta la desaparición de sus siglas en 2015. ${ }^{34}$

\section{Bibliografía}

ALIANZA SOCIALISTA DE ANDALUCÍA (1973), Manifiesto Fundacional. Cuadernos de Ruedo Ibérico, 41-42, 95-101.

ARCHILES, Ferrán (2015), Nacionalismos y culturas políticas en España (1975-2012). En PÉREZ LEDESMA, M. y SAZ, Ismael (Coords.). Del Franquismo a la democracia, 1936-2013. Madrid: Marcial Pons, 141-174.

BARÓN, Enrique (1976). Federación de Partidos Socialistas. Barcelona: Avante.

BOBILLO, Francisco (1976). PSP. Partido Socialista Popular. Barcelona: Avante.

BOYD, Carolyn P. (2000). Historia patria. Política, historia e identidad nacional en España: 1875-1975. Barcelona: Pomares.

CARO CANCELA, Diego (2013). Cien años de socialismo en Andalucía (1885-1985). Cádiz: Quorum.

CARO CANCELA, Diego (2015). La reconstrucción del PSOE en la Andalucia occidental: entre la memoria histórica y la renovación (1975-1982). Historia y Presente, 26, 11-26.

CARRILLO, Santiago y IBÁRRURI, Dolores (1972). Hacia la libertad. VIII Congreso del PCE. París: Editions Sociales, 44-46.

CRUZ ARTACHO, Salvador (2017). ¡Libertad, Amnistía, Democracia y Autonomía! De la memoria dormida a la lucha y conquista de la Autonomía Política para Andalucía

33 Aún hoy se sigue especulando con lo sucedido. El reputado periodista Pepe Fernández años después apunta incógnitas sobre el tema: <https://www.vozpopuli.com/ opinion/chale-politica-andalucia-Escuredo-Junta-Guerra-Gonzalez_0_1137486561.html>

${ }^{34}$ Así se defiende en Ruiz Romero (2004 b). En concreto, a mediados de 1982 los sectores más moderados del Partido del Trabajo de Andalucía se integran en el andalucismo político bajo las siglas PSA-PA. (Santos Silva: 2014) Por otra parte, la I Asamblea del Frente Andaluz de Liberación (FAL) tiene lugar en Sevilla en septiembre de 1979 a partir de militantes del Partido Socialista Unificado de Andalucía (PSUA) con disidentes de PSA, PTA y PCE. Aun reivindicando la independencia para Andalucía en repetidas ocasiones, realizan renuncia expresa al uso de la violencia como estrategia política. Sobre esta efímera opción independentista Cfr. (Stallaert, 1999). 
en la Transición Democrática, 1939-1981. Sevilla: Fundación Centro de Estudios Andaluces.

DE LA FUENTE, Juan José (2017). La 'invención' del socialismo. Radicalismo y renovación en el PSOE durante la dictadura y transición a la democracia (1956-1982). Gijón: Trea.

FEDERACIÓN SOCIALISTA DE ANDALUCÍA-PSOE (1979). Memoria de Gestión. II Congreso, Sevilla.

GARCÍA RUIZ, Carmen Rosa (2001). Planteamientos políticos e ideológicos en las propuestas descentralizadoras o autonómicas de los albores de la transición. Actas del IX Congreso sobre el Andalucismo Histórico. Sevilla: Fundación Blas Infante, 379-380.

GENIOLA, Andrea (2017). Nación, Nacionalidades y Estado. Significación y papel de la cuestión nacional en el socialismo español en (la) Transición (1974-1982). En GONZALEZ MADRID, Damian A., ORTIZ HERAS, Manuel y PÉREZ GARZON, Juan Sisinio (Eds.). La Historia, los in translation? Actas del XIII Congreso de la Asociación de Historia Contemporánea. Cuenca: Universidad de Castilla-La Mancha, 1521-1533.

GILLESPIE, Richard (1991). Historia del Partido Socialista Obrero Español. Madrid: Alianza.

HIJANO DEL RÍO, Manuel y RUIZ ROMERO, Manuel (2001). Documentos para la historia de la autonomía andaluza (1882-1982). Málaga: Sarriá.

LINDE PANIAGUA, Enrique, y HERRERO LERA, Miguel (1980). Comentario a la Ley Orgánica de Modalidades de Referéndum. Revista del Departamento de Derecho Político, 6, 83-105.

LÓPEZ, Victorino (2011). La cuestión territorial en la historia del socialismo español: debate teórico y estrategias políticas (1879-2011). Tesis doctoral Inédita. Madrid: Complutense.

MATEOS, Abdón (1996). Una transición dentro de la transición. Auge, unidad y 'conversión de los socialistas'. En TUSELL, J. y SOTO, A. (eds.). Historia de la Transición, 1975-1986. Madrid: Alianza, 216-235.

MATEOS, Abdón (2017). Historia del PSOE en transición. De la renovación a la crisis. Madrid: Sílex.

MÉNDEZ LAGO, Mónica (2000). La estrategia organizativa del Partido Socialista Obrero Español (1975-1996). Madrid: CIS-Siglo XXI.

MOLINA, J. Daniel (2015). La España del pueblo. La idea de España en el PSOE. Desde la Guerra Civil hasta 1992. Madrid: Silex.

ORTIZ HERAS, Manuel (2009). Culturas políticas del nacionalismo español: del franquismo a la transición. Madrid: Los Libros de la Catarata.

PÉREZ TRUJILLANO, Rubén (2017). Andalucía y reforma constitucional. Córdoba: Almuzara.

PSP (1977). Por un socialismo responsable. El Partido Socialista Popular ante el futuro español. Madrid: Túcar. 
RIPOLLÉS SERRANO, Ma . Rosa (1980). Notas acerca de la Ley Orgánica sobre regulación de las distintas modalidades de referéndum. Comentarios sobre la Constitución española de 1978. Valencia: Universidad, 313.

RODRÍGUEZ DE LA BORBOLLA, José (1986). Andalucía en marcha, Granada: Andalucía.

RODRÍGUEZ-FLORES PARRA, Vega (2017). Nación española y Estado Autonómico en el Partido Socialista Obrero Español (1974-1982), Tesis Doctoral inédita. Valencia: Universidad.

RUBIO RUBIO, Ma . Amalia (1996). Un partido en la oposición (PSP). Granada: Comares. RUIZ ROBLEDO, Agustín (1988). La Comunidad Autónoma de Andalucía. Sevilla: Parlamento de Andalucía.

RUIZ ROBLEDO, Agustín (2003). La formación de la Comunidad Autónoma de Andalucía en sus documentos, Parlamento de Andalucía/Granada: Comares.

RUIZ ROMERO, Manuel (2000a). Política y Administración Pública en el primer Gobierno Preautonómico de Andalucía. La gestión de Plácido Fernández Viagas al frente del ente preautonómico. Sevilla: IAAP.

RUIZ ROMERO, Manuel (2000b). Andalucía Libre. Una revista andaluza de la transición. Índice bibliográfico. Sevilla: Universidad-Grupo de Investigación Estructura, Historia y Contenidos de la Comunicación.

RUIZ ROMERO, Manuel (2002). La emergencia del andalucismo político en el contexto del tardofranquismo a la transición. Actas del III Simposio de historia Actual. Logroño: Instituto de Estudios Riojanos, 639-656.

RUIZ ROMERO, Manuel (2003). El PSOE en Andalucía al inicio de la transición. De la FSA-PSOE al escuredismo (1976-1979). Actas del III Congreso de Historia de Andalucía. Andalucía Contemporánea, III, Córdoba: CajaSur, 591-608.

RUIZ ROMERO, Manuel (2004a). El referéndum para la ratificación autonómica de Andalucía: el 28F como batalla mediática. Sevilla: Fundación Centro de Estudios Andaluces. <http://public.centrodeestudiosandaluces.es/pdfs/H200403.pdf>

RUIZ ROMERO, Manuel (2004 b). El sistema de partidos en la Andalucía de la transición: el fracaso de un discurso diferencial. Actas del VIII Congreso de la Asociación de Historia Contemporánea. Santiago de Compostela: Universidad (formato CD)

RUIZ ROMERO, Manuel (2005). La conquista de la autonomía andaluza (1975-1982). Sevilla: IAAP.

RUIZ ROMERO, Manuel (2008). Tiempos de cambio: Andalucía hacia la Transición autonómica. Sociedad, partidos políticos e instituciones. Sevilla: Ateneo de Sevilla-Universidad.

SÁNCHEZ, José (1979). Las elecciones del 1 de marzo de 1979, un éxito regionalista. Revista de Estudios Regionales, 3, 77-118.

SANTOS, Juliá (1996). Los socialistas en la política española. Madrid: Taurus.

SANTOS LÓPEZ, José Ma . de los (2002). La Andalucía de la Transición. 1976-1982. Sevilla: Fundación Centro de Estudios Andaluces. 
SANTOS SILVA, Alejandro (2014). El papel del Partido del Trabajo de España en la lucha por la autonomía de Andalucía. Historia del presente, 24, 113-125.

SAZ, Ismael y ARCHILÉS, Ferrán (2011). La nación de los españoles. Discursos y prácticas del nacionalismo español en la época contemporánea. Valencia: Universidad.

SOTO FERNÁNDEZ, David, VILLA, Inmaculada, INFANTE, Juan y JAÉN, Santiago (2015). La identidad andaluza como identidad cívica durante la construcción de la autonomía (1975-1982). Sevilla: Fundación Centro de Estudios Andaluces.

STALLAERT, Christiane (1999). La vía andalusí en la encrucijada del etno-andalucismo: El caso de Liberación Andaluza. Actas del VIII Congreso sobre el Andalucismo Histórico. Sevilla: Fundación Blas Infante, 497-510.

TEBA, Juan (1981), La Sevilla de Rojas Marcos, Barcelona, Planeta, 306 a 310.

TEZANOS, José Félix (2003). 125 años del Partido Socialista Obrero Español. Madrid: Fundación Pablo Iglesias. 